CARDIOVASCULAR MEDICINE

\title{
Pathological effects of alcohol septal ablation for hypertrophic obstructive cardiomyopathy
}

\author{
A L Baggish, R N Smith, I Palacios, G J Vlahakes, D M Yoerger, M H Picard, P A Lowry, \\ I-k Jang, M A Fifer
}

See end of article for authors' affiliations

.....................

Correspondence to: M A Fifer, Cardiology Division, Massachusetts General Hospital, 55 Fruit Street, Gray/Bigelow Building, Suite 800 , Mailstop 843, Boston, MA 02114-2696, USA; mfifer@partners.org

Accepted 12 June 2006 Published Online First 28 June 2006

\begin{abstract}
Background: The pathological effects and the mechanisms of action of intracoronary administration of ethanol for alcohol septal ablation (ASA) for the management of hypertrophic obstructive cardiomyopathy (HOCM) are unknown.

Methods: We examined surgical specimens and, in one case, autopsy specimens from four patients who underwent surgical septal myectomy 2 days to 14 months after unsuccessful ASA.

Results: Pathological examination early after ASA showed coagulative necrosis of both the myocardium and the septal perforator arteries. Affected arteries were distended and occluded by necrotic intraluminal debris, without platelet-fibrin thrombi. Late after unsuccessful ASA, excised septal tissue was heterogeneous, containing a region of dense scar, and adjacent tissue containing viable myocytes and interspersed scar.

Conclusions: Intracoronary administration of ethanol in patients with HOCM causes acute myocardial infarction with vascular necrosis. The coagulative necrosis of the arteries, their distension by necrotic debris and the absence of platelet-fibrin thrombi distinguish ethanol-induced infarction from that caused by atherosclerotic coronary artery disease. The direct vascular toxicity of ethanol may be an important aspect of the mechanism of successful ASA.
\end{abstract}

$\mathrm{H}$ ypertrophic cardiomyopathy is a genetic disease characterised by idiopathic thickening of the left ventricle. Pathological evaluation of the myocardium shows myocyte hypertrophy, myocyte disarray and interstitial fibrosis. About $25 \%$ of patients with hypertrophic cardiomyopathy have hypertrophic obstructive cardiomyopathy (HOCM), characterised by dynamic left ventricular outflow tract (LVOT) obstruction due to apposition of an asymmetrically hypertrophied septum with the anterior leaflet of the mitral valve. In most cases, the symptoms of the disease can be controlled by medical treatment. For patients with HOCM and symptoms refractory to optimal medical treatment, surgical septal myectomy or alcohol septal ablation (ASA), in which ethanol is injected into the septum via the coronary circulation, may be considered. ${ }^{1-3}$

Most patients who undergo ASA experience relief of symptoms after the procedure. ${ }^{45}$ High immediate postablation LVOT gradient and low post-ablation peak creatine kinase are predictive of failure of ASA. ${ }^{6}$ Patients who do not benefit from the procedure may undergo repeat ASA, if technically feasible, or surgical septal myectomy. Septal myectomy after ASA affords the opportunity to assess the pathological effects of intracoronary administration of ethanol. Despite the accumulating experience with successful and unsuccessful ASA, reports of the pathological findings after ablation are sparse. To date, the pathology of ASA has not been correlated with clinical information. We report clinicopathological data for patients undergoing ASA followed by surgical myectomy.

\section{PATIENTS AND METHODS}

\section{Selection criteria for ASA}

Selection criteria for ASA were (1) symptoms that interfere substantially with quality of life despite optimal medical treatment; (2) septal thickness $\geqslant 16 \mathrm{~mm}$; (3) LVOT gradient $\geqslant 30 \mathrm{~mm} \mathrm{Hg}$ at rest or $\geqslant 50 \mathrm{~mm} \mathrm{Hg}$ with provocation; (4) accessible septal branches, usually of the left anterior descending coronary artery; and (5) absence of major intrinsic abnormality of the mitral valve and of other conditions for which cardiac surgery is indicated.

\section{ASA procedure and follow-up protocol}

After patients gave informed consent, they were treated with midazolam and fentanyl. Except in patients who had permanent pacemakers, a 4F temporary pacemaker electrode (Bard Electrophysiology, Lowell, Massachusetts, USA) was inserted, usually via the right internal jugular vein, and positioned in the right ventricle. In most cases, left-heart catheterisation was carried out by the trans-septal technique. Heparin $70 \mathrm{U} / \mathrm{kg}$ was given intravenously, and aspirin $324 \mathrm{mg}$ sublingually. In patients with low $(<30 \mathrm{~mm} \mathrm{Hg})$ resting LVOT gradients at the time of ASA, provocation was carried out with dobutamine $5-30 \mu \mathrm{g} / \mathrm{kg} / \mathrm{min}$ (which is more practical and reproducible than exercise in the cardiac catheterisation laboratory).

We operated on the most proximal accessible septal branch using standard angioplasty guiding catheters and guidewires and 1.5 or $2.0 \mathrm{~mm} \times 9 \mathrm{~mm}$ Maverick balloon catheters (Boston Scientific, Natick, Massachusetts, USA). Proper selection of the septal branch and balloon catheter position were confirmed by radiographic and echocardiographic contrast injections. Ethanol labelled as "not less than 98\%" was infused through the balloon catheter at the rate of $1 \mathrm{ml} /$ min. Repeat injections were given in the same or other septal branches, with the goal of reducing the gradient (after balloon deflation) to $<20 \mathrm{~mm} \mathrm{Hg}$. Repeat coronary arteriography was carried out to confirm the absence of normal flow

\footnotetext{
Abbreviations: ASA, alcohol septal ablation; CCS, Canadian Cardiovascular Society; HOCM, hypertrophic obstructive cardiomyopathy; LVOT, left ventricular oufflow tract; MVR, mitral valve replacement; NYHA, New York Heart Association; SAM, systolic anterior motion
} 

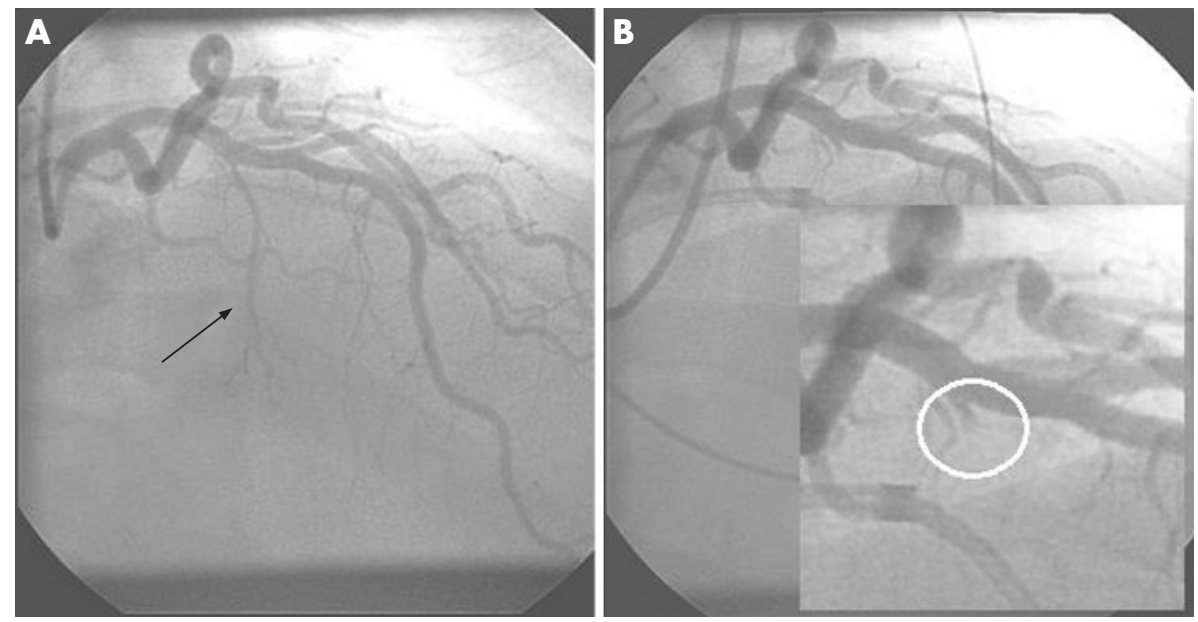

Figure 1 Left coronary arteriogram (A) before and (B) after alcohol septal ablation (ASA). The septal branch (arrow) into which ethanol was injected has a truncated appearance (circle, inset) after ASA.

in the septal branches (fig 1). Patients were managed in the coronary care unit for $\geqslant 1$ day and were transferred to the cardiac step-down unit, with total hospital stay $\geqslant 3$ days. Temporary pacemakers were usually left in place for $\geqslant 2$ days.

Clinical evaluation and echocardiography were repeated 3 months after ASA. We deemed the procedure to have been successful if symptoms were improved by $\geqslant 1$ New York Heart Association (NYHA) or Canadian Cardiovascular Society (CCS) class, and the peak LVOT gradient measured by Doppler echocardiography was reduced by $\geqslant 50 \%$. Patients with unsuccessful primary ASA were offered a repeat percutaneous procedure, if technically possible, or surgical septal myectomy.

\section{Management of surgical specimens}

Myocardial specimens obtained at the time of surgical septal myectomy were fixed in $10 \%$ buffered formalin and embedded in paraffin wax using standard departmental protocol. Multiple histological specimens were examined with haematoxylin-eosin, trichome and elastic stains using standard histological techniques.

\section{Statistics}

Data are shown as mean (standard deviation (SD)). Comparisons between groups were carried out using a twotailed t test with a significance level of 0.05 .

\section{RESULTS}

\section{Patient characteristics}

Between September 1998 and January 2005, we attempted ASA in 64 patients ( 38 women and 26 men). Patients were 57 (SD 16, range, 16-84) years old. Pre-procedure NYHA class was 2 in 13 patients, 3 in 41 and 4 in 10 patients. CCS angina class was 0 in 24 patients, 1 in 13, 2 in 10, 3 in 10 and 4 in 7 patients. The mean (SD) septal thickness was 19 (3) $\mathrm{mm}$ and peak resting or, in 14 cases, provoked LVOT gradient was 83 (35) $\mathrm{mm} \mathrm{Hg}$. With one exception (patient 2), left ventricular systolic function was normal.

\section{Results of ASA}

In 4 of 64 attempted ASA procedures, ethanol was not injected. Of these four patients, two had unfavourable septal anatomy that precluded the delivery of ethanol; in one of these (patient 1), a covered stent was placed, ${ }^{7}$ whereas in the other, pericardial tamponade due to right ventricular perforation by the pacemaker electrode was recognised and corrected, and ethanol administration was deferred. In the remaining patient, ethanol was not administered as coronary dissection was managed successfully with stenting. Complications included coronary artery dissection $(n=2)$, pericardial tamponade $(n=2)$, ventricular tachyarrhythmias requiring countershock $(\mathrm{n}=3)$ and conduction defects for which permanent pacemakers were implanted $(n=11)$. Other than the single death reported in a patient who was moribund before the procedure (patient 2), no patient undergoing ASA has died to date.

Ethanol was infused in $>1$ septal branch in 13 procedures. The mean (SD) total ethanol dosage was 2.9 (SD 1.8) ml. ASA was successful in reducing both symptoms and LVOT gradient in 51 of the $64(80 \%)$ patients. The mean (SD) NYHA class decreased from $3.0(0.6)$ to $1.7(1.0 ; \mathrm{p}<0.001)$ and CCS class from $1.4(1.4)$ to $0.5(1.2 ; \mathrm{p}<0.001)$. The mean peak creatine kinase concentration was 1471 (720) U/l after successful ASA and 1324 (892) U/l after unsuccessful ASA $(p=0.65)$. The mean (SD) LVOT gradient immediately after ablation was 23 (24) after successful ASA and 32 (25) after unsuccessful ASA ( $p=0.42)$.

Surgery or a second ASA procedure was carried out in 12 of the 13 patients with unsuccessful primary ASA. The decision to perform surgery or a second ASA procedure was based on septal perforator anatomy and patient preference. Five patients underwent a second ASA procedure, while the other seven patients underwent surgery, including septal myectomy $(\mathrm{n}=4$, including two patients who did not receive ethanol), mitral valve replacement $(M V R ; n=1)$, combined septal myectomy and MVR $(\mathrm{n}=1)$, and combined septal myectomy and resection of a subaortic membrane $(n=1)$. Cases of four patients who had unsuccessful ethanol infusion, including one patient who underwent covered stent septal ablation, and had subsequent septal myectomy, are described below.

\section{Unfavourable coronary anatomy preventing administration of ethanol \\ Case 1}

A 76-year-old woman with exertional dyspnoea and recurrent syncope had a systolic murmur. Some of her clinical data have been reported previously. ${ }^{7}$ The echocardiogram showed septal thickness $18 \mathrm{~mm}$, posterior wall $13 \mathrm{~mm}$, systolic anterior motion (SAM) of the mitral valve, LVOT gradient $78 \mathrm{~mm} \mathrm{Hg}$ and mild mitral regurgitation. Coronary arteriography showed $20 \%$ stenosis of the left anterior descending artery involving the take-off of the first septal branch, which stood at an acute angle. ASA was unsuccessful, both before 
and after directional atherectomy of the left anterior descending artery, because of the inability to instrument the first septal branch. Ethanol was not given. A covered stent was placed in the left anterior descending artery, totally occluding the first septal branch and producing nausea in the absence of chest discomfort. The LVOT gradient was reduced to $15 \mathrm{~mm} \mathrm{Hg}$. Peak creatine kinase was $363 \mathrm{U} / \mathrm{l}$. No changes were seen on the electrocardiogram. Three months after this covered stent septal ablation, she was free of cardiac symptoms and peak LVOT gradient was $21 \mathrm{~mm} \mathrm{Hg}$.

Six months after septal ablation, the original symptoms recurred. Ten months after ablation, the LVOT gradient was $97 \mathrm{~mm} \mathrm{Hg}$. Coronary arteriography showed collateralisation of the occluded septal branch from the right coronary artery. There were also "candy wrapper" stenoses of the left anterior descending artery at both ends of the covered stent. Septal myectomy and coronary bypass grafting were carried out, with a left internal mammary artery graft placed on the left anterior descending artery. A jet lesion was observed on the septum. Histological examination (fig 2) showed incomplete infarction of the myocardium, with areas of viable-appearing, hypertrophied myocytes (fig 2A) interspersed with areas of scar tissue (fig 2B).

\section{Pathology early after ASA} Case 2

A 68-year-old woman had pulmonary oedema in the setting of the systemic inflammatory response syndrome. The echocardiogram showed septal thickness $20 \mathrm{~mm}$, posterior wall $12 \mathrm{~mm}$, SAM, LVOT gradient $120 \mathrm{~mm} \mathrm{Hg}$, moderate mitral regurgitation, and anteroapical and distal inferior akinesis, indicating combined HOCM and apical ballooning syndrome. ${ }^{8}$ Cardiac catheterisation confirmed the wall motion abnormalities and revealed normal coronary arteries. The patient's clinical status deteriorated further, with hypotension, metabolic acidosis and oliguric renal failure, prompting placement of a left ventricular assist device. Attempts to wean left ventricular support were unsuccessful. ASA was carried out, without reduction in the LVOT gradient despite injection of $3 \mathrm{ml}$ of ethanol into each of the first and second septal branches. Peak creatine kinase was 2014 U/l. Two days later, the patient underwent removal of the left ventricular assist device, surgical septal myectomy and MVR. The septum was grossly necrotic, and had a jet lesion. The LVOT gradient was abolished, but there was persistent postoperative hypotension, resulting in death 1 day after surgery.

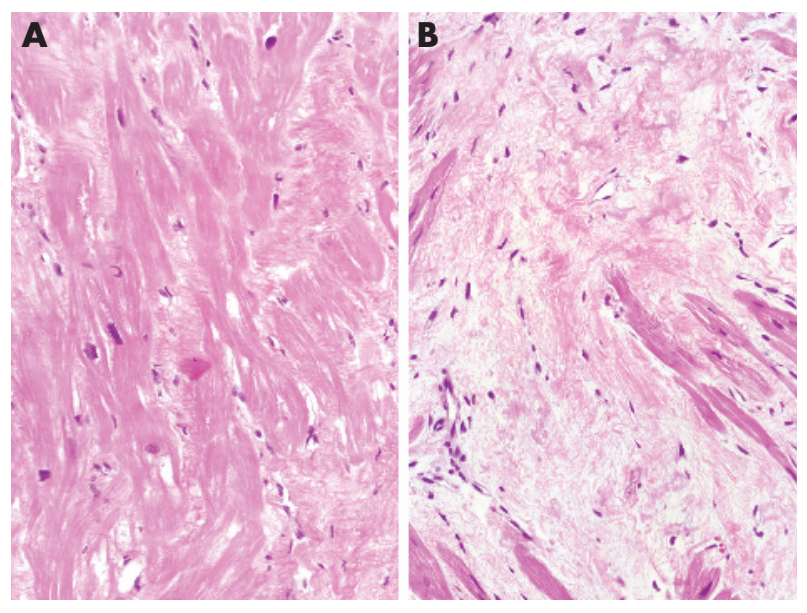

Figure 2 Myocyte hypertrophy with (A) fibre disarray and (B) scar tissue, from a patient who did not receive ethanol but underwent covered stent septal ablation (haematoxylin-eosin, $\times 200$ ).
Figure 3 shows the myectomy specimen. The infarcted tissue was yellow (fig 3A). Microscopical examination showed myocardial necrosis (fig 3B) with marginating neutrophils at the periphery of the infarction (fig 3C,D). The autopsy specimen weighed $540 \mathrm{~g}$ (fig 4). Serial sectioning of the coronary arteries showed no major atherosclerotic disease or thrombosis. A cross-section of the heart (fig 4A) showed necrotic yellow septal myocardium. Microscopical examination showed myocyte hypertrophy and disarray (fig 4B), with ethanol-induced necrosis. Necrosis of the septal perforator artery and adjacent myocardium was seen, with absence of nuclei from endothelial and smooth-muscle cells (fig 4C,D). The artery was distended with necrotic luminal debris (fig 4C,D). Fibrin thrombi were not observed.

\section{Pathology late after ASA \\ Case 3}

A 56-year-old woman had palpitations, angina, exertional dyspnoea and light-headedness, and one episode of syncope. She had a systolic murmur. The echocardiogram showed septal thickness $21 \mathrm{~mm}$, posterior wall $12 \mathrm{~mm}$, SAM, LVOT gradient $74 \mathrm{~mm} \mathrm{Hg}$ and moderate mitral regurgitation. Coronary arteriography was normal. Ethanol, $2 \mathrm{ml}$, was injected into the first septal branch of the left anterior descending artery, with reduction in the LVOT gradient to $20 \mathrm{~mm} \mathrm{Hg}$. Peak creatine kinase was 1305 U/l. The patient's cardiac symptoms persisted. A follow-up echocardiogram at 3 months showed LVOT gradient $137 \mathrm{~mm} \mathrm{Hg}$. Six months after ASA, surgical myectomy was performed. A small septal scar was evident at myectomy, but an adjacent region of the septum, judged intraoperatively to be responsible for outflow tract obstruction, appeared viable. Pathological examination of this tissue showed viable-appearing myocytes intermingled with the scar, indicating incomplete ethanolinduced infarction (fig 5).

\section{Case 4}

An 82-year-old woman with a history of hypertension had progressive exertional dyspnoea and one episode of nearsyncope. She had a systolic murmur. The echocardiogram showed septal thickness $19 \mathrm{~mm}$, posterior wall $12 \mathrm{~mm}$, LVOT gradient $69 \mathrm{~mm} \mathrm{Hg}$, SAM and moderate mitral regurgitation. Coronary arteriography was normal. Ethanol, $1 \mathrm{ml}$, was injected into the first septal branch of the anterior descending artery, with reduction in the gradient to $30 \mathrm{~mm} \mathrm{Hg}$. Peak creatine kinase was $736 \mathrm{U} / \mathrm{l}$. The patient continued to experience exertional dyspnoea. LVOT gradient was $85 \mathrm{~mm}$ $\mathrm{Hg}$ at 3 months and $83 \mathrm{~mm} \mathrm{Hg} 8$ months after ASA. Septal myectomy was performed 14 months after ASA. The excised septal tissue had a focal area of complete fibrosis, but otherwise appeared grossly viable. Examination of the myectomy specimen showed residual non-infarcted myocardium with myocyte hypertrophy and mild disarray (fig 6A). Incomplete infarction was shown by the presence of viableappearing myocytes (red) surrounded by scar (blue). A small septal artery in the region of excised scar tissue was thrombosed (fig 6B). The artery wall was necrotic and lacked viable smooth muscle. An elastic stain (fig $6 \mathrm{C}$ ) verified that the scarred artery still contained some internal elastic lamina.

\section{DISCUSSION}

Ethanol is a potent tissue toxin used in the ablative treatment of hepatocellular and renal cell carcinomas, benign prostatic hypertrophy and vascular malformations. ${ }^{9-12}$ In patients with cardiac disease, ethanol has been used to ablate ventricular tachyarrhythmias. ${ }^{13}$ In patients with HOCM, targeted injection of ethanol into the interventricular septum usually results in reduction in the LVOT gradient, with improvement in symptoms. ${ }^{45}$ Since the publication of the first report of 

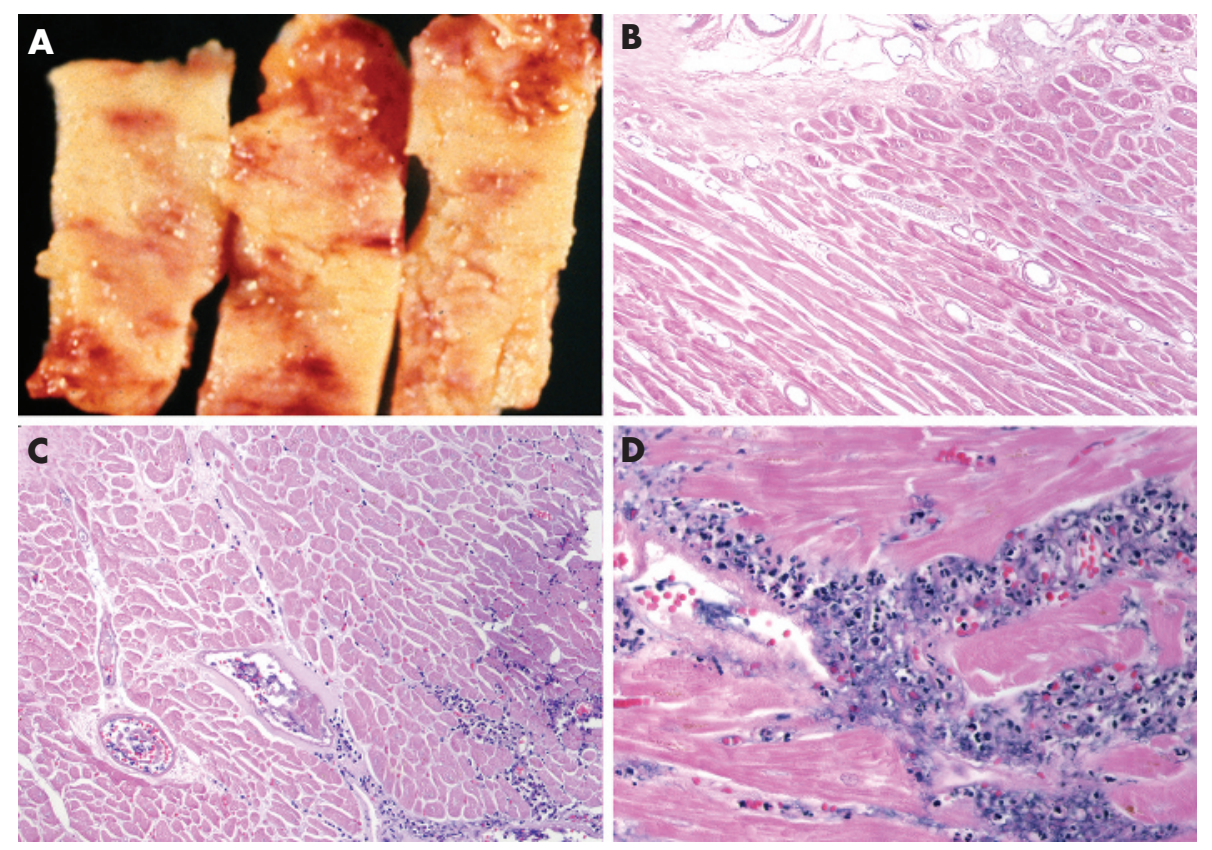

Figure 3 Myectomy specimen 2 days after alcohol septal ablation, $1.2 \times 0.4 \mathrm{~cm}$. (A) Necrotic yellow myocardium. (B) Necrotic myocardium (haematoxylin-eosin $(H \& E), \times 100)$. (C) Necrotic myocardium with marginating neutrophils $(H \& E, \times 100)$. (D) Neutrophils marginating into necrotic tissue $(H \& E, \times 200)$.

ASA in $1995,{ }^{2}$ many procedures have been performed, but there are few pathological data with clinical correlation.

\section{Effects of ethanol injection}

The effects of alcohol on various tissues have been explored in animal studies. Oshita $e t \mathrm{al}^{14}$ observed an acute elevation in portal vein pressure after the infusion of ethanol into the portal veins of rats. The rapid pressure rise was inhibited by nitroprusside, suggesting that the mechanism of pressure increase was ethanol-induced vasospasm. In rats, injection of $70 \%$ ethanol into the carotid artery caused full-thickness necrosis of the vessel wall and swelling of brain cells. ${ }^{15}$ The vascular toxicity of ethanol was also shown in canine basilar arteries, in which ethanol induced dose-dependent endothelial injury, platelet adhesion and fibrin clot formation. ${ }^{16}$

In dogs, intracoronary injection of $100 \%$ ethanol caused myocardial necrosis involving greater than half the thickness of the left ventricular wall. ${ }^{17}$ The resultant necrosis was focal
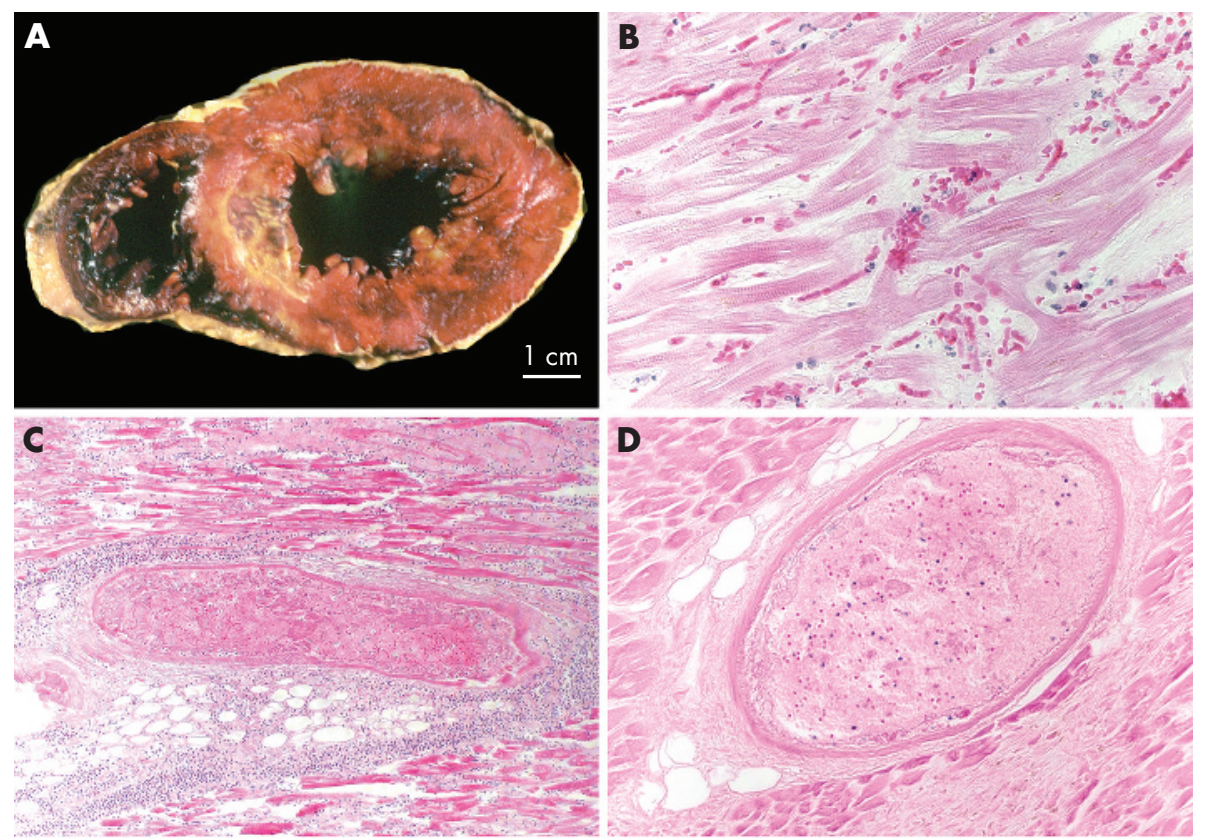

Figure 4 Autopsy specimen 3 days after alcohol septal ablation and 1 day after surgery. (A) Cross-section of heart showing thinned and partially necrotic (yellow) septum. (B) Myocyte hypertrophy, fibre disarray and necrosis (haematoxylin-eosin (H\&E), $\times 200$ ). (C) Section of septal perforator and surrounding myocardium, showing necrosis and marginating neutrophils $(\mathrm{H} \& \mathrm{E}, \times 100)$. (D) Septal perforator artery and surrounding necrotic myocardium $(\mathrm{H} \& \mathrm{E}, \times 200)$; the endothelial and smooth-muscle cells of the artery wall lack nuclei, indicating necrosis. The artery is distended and occluded by necrotic debris. 


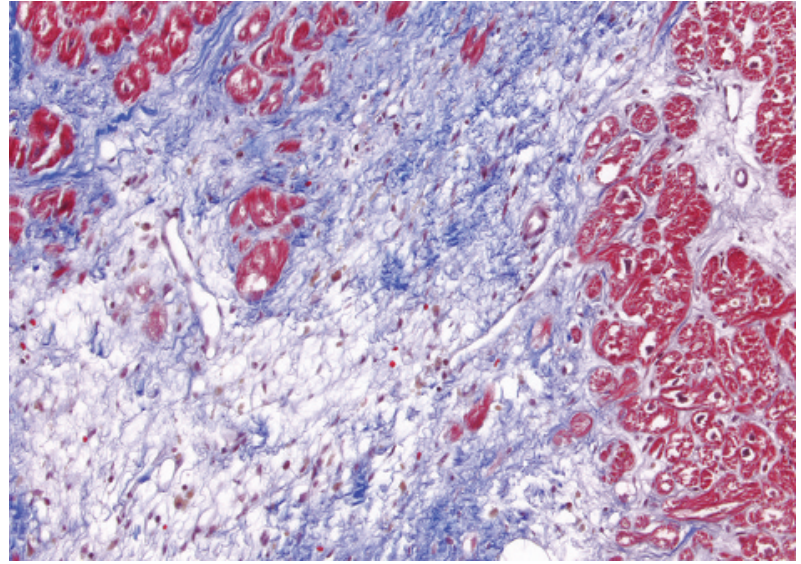

Figure 5 Hypertrophied myocytes interspersed with scar tissue, 6 months after alcohol septal ablation (trichrome stain, $\times 200$ ).

or patchy and non-confluent. Intraluminal fibrin or thrombus was present in the operated coronary branch in three of eight dogs. Inoue et $a l^{17}$ speculated that ethanol may have induced thrombus formation even in the absence of intimal injury. Injection of lower concentrations of ethanol caused similar patterns of necrosis without intraluminal clot. Injection of $100 \%$ ethanol into the canine coronary venous circulation caused myocardial necrosis. ${ }^{18}$ Transventricular injection of $60 \%$ ethanol in dogs produced coagulative necrosis and haemorrhage, with a narrow border zone consisting of myocytes with neutrophilic infiltration. ${ }^{19}$ After 1 month, there was dense fibrosis with mild to moderate inflammation. Weismüller $e t \mathrm{al}^{20}$ injected ethanol directly into canine myocardium through a catheter system in the left ventricular cavity. Animals were killed 34 weeks after ethanol injection. The investigators observed myocardial necrosis but no intraluminal thrombosis.

Relatively few human pathological data are available on ethanol-induced myocardial infarction in the context of septal ablation. Arrazaghi et al ${ }^{21}$ observed replacement fibrosis with islands of myocytes in a myectomy specimen obtained $21 / 2$ years after ASA. ${ }^{21}$ Raute-Kreinsen ${ }^{22}$ examined the hearts of 13 patients who died between $25 \mathrm{~min}$ and 2 years after ASA; 10 of the deaths were within 9 days of the procedure. Few clinical data were provided. Macroscopic and microscopic signs of necrosis appeared between $25 \mathrm{~min}$ and $11 \mathrm{~h}$. In the patient who died $25 \mathrm{~min}$ after ASA, blood in the coronary lumen was "lumpily fixed". At $11 \mathrm{~h}$, the myocardium seemed to be "vitally fixed". A microscopic scar formed between 9 days and 1 month. Raute-Kreinsen concluded that ethanol "fixes the tissue in its natural structure"; leucocytes are attracted but are unable to penetrate the fixed tissue, so that cell lysis occurs only in the border zone. Our pathological findings are in agreement with those of Raute-Kreinsen. ${ }^{22}$ Ethanol causes coagulative necrosis of both the myocardium and the arteries, with marginating neutrophils at the periphery of the infarct. Late after ablation, necrotic myocardium is replaced by a permanent scar and granulation tissue interspersed with islands of atrophic myocardium.

Early after exposure of the myocardium to ethanol, we observed coagulative necrosis with intense leucocytic infiltration and tissue oedema, similar to that observed in spontaneous myocardial infarction associated with coronary artery disease. In distinction to the pathology of spontaneous myocardial infarction, however, the septal branches showed endothelial and smooth-muscle necrosis, and luminal occlusion with necrotic debris and "fixed" blood. Fixation of blood and direct toxicity to the vessel wall may be unique
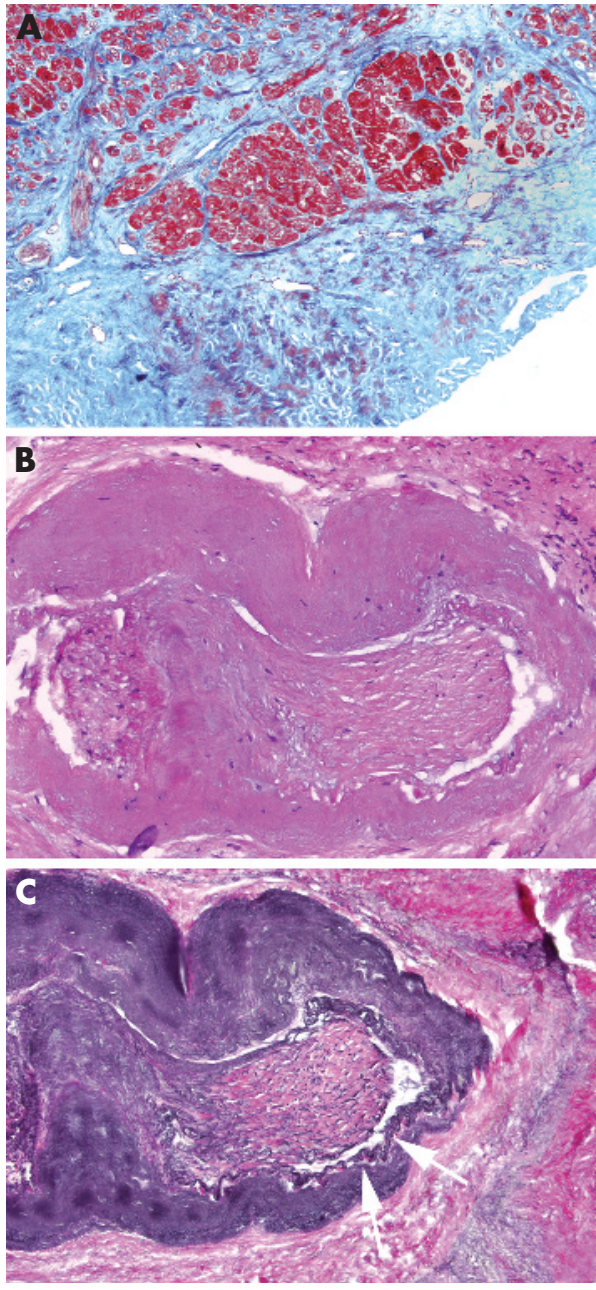

Figure 6 Myectomy specimen, 14 months after alcohol septal ablation. (A) Viable myocytes (red) surrounded by scar (blue; trichrome stain, $\times 200$ ). (B) Necrotic vessel wall containing no visible nuclei, with thrombosis of vessel lumen (haematoxylin-eosin, $\times 100$ ). (C) Thrombosed necrotic small artery mummified by ethanol, showing residual internal elastic lamina (white arrows; elastic stain, $\times 100$ ).

components of ethanol-induced tissue injury. The intraluminal occlusion associated with ethanol injection may also cause an ischaemic infarction distal to the occlusion. Ultimately, much of the necrotic myocardium is eventually replaced by scar tissue, as occurs after spontaneous myocardial infarction.

Thus, in addition to its previously proved toxicity to myocardium, ethanol may be toxic to coronary arteries. In patients undergoing ASA, it is not clear what component of myocardial necrosis results from ischaemia caused by coronary occlusion as opposed to direct toxicity of ethanol. Immediately after ASA, coronary arteriography shows a lack of perfusion of the septal territory in which ethanol has been injected ("no reflow" phenomenon). The toxicity of ethanol to the vasculature, including the microvasculature, may preclude myocardial protection by collateral blood flow, ensuring the efficacy of ethanol in causing myocardial necrosis. When antegrade blood flow is interrupted but ethanol is not given (case 1), myocardial necrosis may be limited by collateral blood flow, ultimately rendering ASA unsuccessful.

\section{Reasons for unsuccessful ASA}

Factors shown to predict failure of ASA are immediate postablation LVOT gradient and low post-ablation peak creatine 
kinase. ${ }^{6}$ For our patients, trends for both of these findings did not reach statistical significance, although our study was not powered to tackle these issues. Failure may occur when the full region of the septum responsible for outflow tract obstruction is not exposed to ethanol (cases 3 and 4). Incomplete ablation of the septum may be related to the distribution or selection of septal vessels or to ethanol dosage. In a patient with HOCM complicated by shock and apical ballooning syndrome (case 2), preload and afterload reduction secondary to sepsis and hyperdynamic septal contraction associated with extensive anteroapical and inferoapical wall motion abnormality, which may produce an LVOT gradient even in the absence of hypertrophy, may also preclude effective gradient reduction by ASA. ${ }^{23}$

\section{Limitations of the present study}

With one exception (case 2), no patients at the Massachusetts General Hospital, Boston, have died after ASA. Our study population is therefore limited to patients undergoing septal myectomy after failed ASA, and is consequently small. Furthermore, there is obvious selection bias introduced by examining specimens only from patients with failed ASA procedures. Autopsy studies on patients who ultimately die after successful ASA would be helpful in resolving this issue.

\section{CONCLUSIONS}

In addition to toxicity to and resultant necrosis of myocardium, ethanol also causes necrosis of coronary arteries. After intracoronary injection of ethanol, coagulative necrosis of the injected septal branches occurs, distinguishing this form of infarction from that attributable to atherosclerotic coronary artery disease. The direct vascular toxicity of ethanol may be important to the mechanism of successful ASA.

\section{Authors' affiliations}

A L Baggish, I Palacios, D M Yoerger, M H Picard, P A Lowry, I-k Jang, M A Fifer, Department of Medicine, Massachusetts General Hospital, Boston, Massachusetts, USA

R N Smith, Department of Pathology, Massachusetts General Hospital, Boston, Massachusetts, USA

G J Vlahakes, Department of Surgery, Massachusetts General Hospital, Boston, Massachusetts, USA

Competing interests: None declared.

\section{REFERENCES}

1 Morrow AG, Fogarty TJ, Hannah H 3rd, et al. Operative treatment in idiopathic hypertrophic subaortic stenosis. Techniques, and the results of preoperative and postoperative clinical and hemodynamic assessments. Circulation 1968;37:589-96.
2 Sigwart U. Non-surgical myocardial reduction for hypertrophic obstructive cardiomyopathy. Lancet 1995;346:21 1-14.

3 Seggewiss H, Faber L, Gleichmann U. Percutaneous transluminal septal ablation in hypertrophic obstructive cardiomyopathy. Thorac Cardiovasc Surg 1999;47:94-100.

4 Gietzen FH, Leuner CJ, Raute-Kreinsen U, et al. Acute and long-term results after transcoronary ablation of septal hypertrophy (TASH). Catheter interventional treatment for hypertrophic obstructive cardiomyopathy. Eur Heart J 1999;20:1342-54.

5 Lakkis NM, Nagueh SF, Dunn JK, et al. Nonsurgical septal reduction therapy for hypertrophic obstructive cardiomyopathy: one-year follow-up. J Am Coll Cardiol 2000;36:852-5.

6 Chang SM, Lakkis NM, Franklin J, et al. Predictors of outcome after alcohol septal ablation therapy in patients with hypertrophic obstructive cardiomyopathy. Circulation 2004;109:824-7.

7 Fifer MA, Yoerger DM, Picard MH, et al. Images in cardiovascular medicine. Covered stent septal ablation for hypertrophic obstructive cardiomyopathy: initial success but ultimate failure resulting from collateral formation, Circulation 2003;107:3248-9.

8 Tsuchihashi K, Ueshima K, Uchida T, et al. Transient left ventricular apical ballooning without coronary artery stenosis: a novel heart syndrome mimicking acute myocardial infarction. Angina Pectoris-Myocardial Infarction Investigations in Japan. J Am Coll Cardiol 2001;38:11-18.

9 Lee BB, Do YS, Byun HS, et al. Advanced management of venous malformation with ethanol sclerotherapy: mid-term results. J Vasc Surg 2003;37:533-8.

10 Munro NP, Woodhams S, Nawrocki JD, et al. The role of transarteria embolization in the treatment of renal cell carcinoma. BJU Int 2003:92:240-4

11 Huo TI, Huang YH, Wu JC, et al. Comparison of percutaneous acetic acid injection and percutaneous ethanol injection for hepatocellular carcinoma in cirrhotic patients: a prospective study. Scand J Gastroenterol 2003:38:770-8.

12 Chiang PH, Chuang YC, Huang CC, et al. Pilot study of transperineal injection of dehydrated ethanol in the treatment of prostatic obstruction. Urology 2003;61:797-801.

13 Brugada P, de Swart H, Smeets IL, et al. Transcoronary chemical ablation of ventricular tachycardia. Circulation 1989;79:475-82.

14 Oshita M, Sato N, Yoshihara $\mathrm{H}$, et al. Ethanol-induced vasoconstriction causes focal hepatocellular injury in the isolated perfused rat liver. Hepatology 1992;16:1007-13.

15 Sampei K, Hashimoto N, Kazekawa K, et al. Histological changes in brain tissue and vasculature after intracarotid infusion of organic solvents in rats. Neuroradiology 1996;38:291-4.

16 Kobayashi $\mathbf{H}$, Ide $\mathrm{H}$, Kabuto $\mathrm{M}$, et al. Endothelial-cell injury of the basilar artery caused by ethanol infusion in dogs. Acta Neurochir (Wien) 1996; 138:84-9.

17 Inove H, Waller BF, Zipes DP. Intracoronary ethyl alcohol or phenol injection ablates aconitine-induced ventricular tachycardia in dogs. J Am Coll Cardiol 1987; 10:1342-9.

18 Wright KN, Morley T, Bicknell J, et al. Retrograde coronary venous infusion of ethanol for ablation of canine ventricular myocardium. J Cardiovasc Electrophysiol 1998;9:976-84.

19 Goette A, Hartung W, Lesh M, et al. Transcatheter subendocardial infusion. A novel technique for mapping and ablation of ventricular myocardium. Circulation 1996:94:1449-55.

20 Weismüller $\mathbf{P}$, Mayer U, Richter $\mathrm{P}$, et al. Chemical ablation by subendocardial injection of ethanol via catheter-preliminary results in the pig heart. Eur Heart J 1991; 12:1234-9

21 Arrazaghi AA, Butany JW, Williams WG, et al. Myectomy for hypertrophic obstructive cardiomyopathy after failed alcohol septal ablation: clinicopathological correlations. Can J Cardiol 2001;17:197-202.

22 Raute-Kreinsen U. Morphology of necrosis and repair after transcoronary ethanol ablation of septal hypertrophy. Pathol Res Pract 2003;199:121-7.

23 Villareal RP, Achari A, Wilansky S, et al. Anteroapical stunning and left ventricular outflow tract obstruction. Mayo Clin Proc 2001;76:79-83. 